April 2012

\title{
Short course adjuvant terlipressin in acute variceal bleeding: A randomized double blind dummy controlled trial
}

Zahid Azam

Aga Khan University

Saeed Hamid

Aga Khan University

Wasim Jafri

Aga Khan University

Mohammad Salih

Aga Khan University

Zaigham Abbas

Aga Khan University

See next page for additional authors

Follow this and additional works at: https://ecommons.aku.edu/

pakistan_fhs_mc_med_gastroenterol

Part of the Gastroenterology Commons

\section{Recommended Citation}

Azam, Z., Hamid, S., Jafri, W., Salih, M., Abbas, Z., Abid, S., Shah, H. (2012). Short course adjuvant terlipressin in acute variceal bleeding: A randomized double blind dummy controlled trial. Journal of Hepatology, 56(4), 819-824.

Available at: https://ecommons.aku.edu/pakistan_fhs_mc_med_gastroenterol/31 
Authors

Zahid Azam, Saeed Hamid, Wasim Jafri, Mohammad Salih, Zaigham Abbas, Shahab Abid, and Hasnain Shah 


\title{
Short course adjuvant terlipressin in acute variceal bleeding: A randomized double blind dummy controlled trial
}

\author{
Zahid Azam ${ }^{1,2}$, Saeed Hamid ${ }^{1, *}$, Wasim Jafri ${ }^{1}$, Mohammad Salih ${ }^{1}$, Zaigham Abbas ${ }^{1}$, \\ Shahab Abid ${ }^{1}$, Hasnain Shah ${ }^{1}$ \\ ${ }^{1}$ Section of Gastroenterology, Department of Medicine, The Aga Khan University, Karachi, Pakistan; ${ }^{2}$ National Institute of Liver \\ $\mathcal{E}$ Gastro-intestinal Diseases (NILGID), Dow University of Health Sciences, Karachi, Pakistan
}

\begin{abstract}
Background \& Aims: Terlipressin is recommended for 3-5 days as adjuvant to endoscopic variceal band ligation (EVBL) in esophageal variceal bleeding (EVB). We assessed whether terlipressin can be administered for a shorter period of time to patients with EVB.

Methods: All eligible EVB patients received $24 \mathrm{~h}$ of open label terlipressin at presentation. After successful EVBL, patients were randomized to receive active or dummy terlipressin for the next $48 \mathrm{~h}$. We excluded patients with failure to achieve initial hemostasis, bleeding gastric varices, known hepatoma, and/or portal vein thrombosis, advanced cirrhosis (Child-Pugh score $\geqslant 12$ ), and patients on a ventilator. The primary outcome was failure to control EVB. The secondary outcomes were 30-day mortality; re-bleeding and composite outcome of failure to control EVB.
\end{abstract}

Results: A total of 130 eligible patients were randomized to receive terlipressin for a total of 24 (short course or SC) or $72 \mathrm{~h}$ (usual course or UC). Baseline patient characteristics were comparable; the majority of patients were $\mathrm{HCV}$-infected and male. There was one failure to control EVB (1.5\%) in UC and none in SC terlipressin $(p=0.50)$. The 30 -day re-bleeding rate was $1.5 \%$ and $3.1 \%$ in UC, and SC terlipressin, respectively $(p=0.50)$. The 30-day mortality was $12,6(9.2 \%)$ patients in each group $(p=0.50)$. The 30-day failure to control bleeding was observed in 14 patients; seven in each group $(p=0.494)$.

Conclusions: In patients with esophageal variceal bleeding, a $24-$ $\mathrm{h}$ course of terlipressin is as effective as a 72-h course when used as an adjunctive therapy to successful EVBL.

(c) 2011 European Association for the Study of the Liver. Published by Elsevier B.V. All rights reserved.

Keywords: Portal hypertension; Variceal hemorrhage; Gastrointestinal hemorrhage; Cirrhosis; Terlipressin; Randomized controlled trial; Dummy; Outcome study.

Received 10 July 2011; received in revised form 3 November 2011; accepted 3 November 2011; available online 16 December 2011

* Corresponding author. Address: Department of Medicine, The Aga Khan University, Stadium Road, Karachi, Pakistan. Tel.: +92 21 34864661; fax: +92 21 34934294.

E-mail address: saeed.hamid@aku.edu (S. Hamid).

Abbreviations: EVBL, esophageal variceal band ligation; EVB, esophageal variceal bleed; SC, short course; UC, usual course; HCV, hepatitis C Virus; PVT, portal vein thrombosis; HCC, hepatocellular carcinoma; GCP, good clinical practice; ER, emergency room; GV, gastric varix; EV, esophageal varix; EKG, electrocardiogram; $\mathrm{BCU}$, bleeding care unit; $\mathrm{Hb}$, hemoglobin; ITT, intention to treat; $\mathrm{CI}$, confidence interval; IHD, ischemic heart disease; CPS, Child-Pugh Score.

\section{Introduction}

Bleeding from gastro-esophageal varices is an important and severe complication of progressive liver disease. Varices are identified in about $30 \%$ of patients with compensated cirrhosis and $60 \%$ of patients with de-compensated cirrhosis [1]. Esophageal variceal bleeding (EVB) occurs in $10-20 \%$ of cirrhotic patients per year and each bleeding episode can be associated with inhospital mortality [2]. EVB remains the major cause of death in these patients, although the mortality has decreased substantially in the last 20 years [3-4]. Currently, the keystone of therapy of acute EVB is endoscopic variceal band ligation (EVBL). In addition, vasoactive agents like terlipressin, octreotide, vapreotide or somatostatin are recommended to be started as adjunctive therapy [5] and found to be highly effective in the management of these patients. Furthermore, these vasoactive drugs are recommended to be continued for $72-120 \mathrm{~h}$ (3-5 days) by various guidelines, including Baveno IV [5], as the risk of re-bleeding is considered to be the highest in the first 5 days of index bleed [6].

The use of terlipressin as an adjunctive therapy has received recent attention, and was found to be associated with survival benefits in a meta-analysis compared with the endoscopic intervention alone or other vasoactive agents [7-9]. In a randomized controlled trial from our group reported earlier [10], terlipressin was found to be of comparable efficacy to octreotide, along with endoscopic intervention, for the control of variceal bleeding.

Although recommended for 3-5 days, the optimal duration of vasoactive drug use in an individual patient remains an unsettled question. One reason may be that these drugs have not been well studied in clinical situations for a short duration, especially when the risk of re-bleeding could be considered to be low. For example, patients with active bleeding at endoscopy or failure to control initial hemostasis, portal vein thrombosis (PVT), hepatocellular carcinoma (HCC), high bilirubin levels, high Child-Pugh score are considered at high risk for poor outcome for re-bleeding and mortality [2] compared to patients where these factors are not present. In our clinical practice, we have observed that shortening the duration of terlipressin to $24 \mathrm{~h}$ in selected patients with variceal bleeding, in which good hemostasis had been achieved with EVBL, did not adversely affect clinical outcomes [11]. Therefore, in the present study, we have prospectively tested the hypothesis that $24 \mathrm{~h}$ of terlipressin treatment (short course or $\mathrm{SC}$ ) is non-inferior to $72 \mathrm{~h}$ of terlipressin treatment (usual course

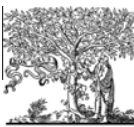

Journal of Hepatology 2012 vol. 56 | 819-824

ELSEVIER 


\section{Research Article}

or UC) in EVB patients after successful EVBL. If proven correct, this could have important implications on the cost of managing acute variceal bleeding and may also decrease the side effects of therapy.

\section{Materials and methods}

Study design

From August 2006 until September 2008, we conducted an investigator initiated randomized, double blind, dummy controlled trial of short $v s$. usual course of terlipressin as an adjuvant to EBVL in patients presenting with EVB, in our center. The study was approved by the Ethical Review Committee (ERC) of our university; an informed consent was obtained from each study subject or his/her next of kin in case the study subject was incompetent to give consent, and the study was conducted following the Good Clinical Practice (GCP) guidelines. An independent data safety monitoring board monitored the trial and had access to non-blinded data, and the study protocol was registered at www.clinicaltrials.gov (ClinicalTrials.gov identifier: NCT00369694).

\section{Study patients}

The study subjects were adult cirrhotic patients presenting to the emergency room (ER) with a history of hematemesis (frank blood or coffee ground emesis) and/or melena (black tarry stools). They were either known cirrhotics or, in some patients, cirrhosis was diagnosed on the basis of clinical examination, laboratory investigation results, and/or ultrasonography. Esophageal variceal bleeding was diagnosed if, at endoscopy, active bleeding was seen from a varix or patients presented with red color signs on their esophageal varices with blood in the esophagus or stomach and no other potential site of bleeding was identified. In the presence of both gastric varix (GV) and esophageal varices (EV), EVB was considered if EV were large ( $\geqslant 5 \mathrm{~mm}$ in diameter), no other cause of acute bleeding was identified and no stigmata of recent bleeding were noted on GV (red sign or a punctum).

We excluded patients who were potentially at high risk of re-bleeding, defined as initial failure to control EVB with EVBL, the presence of known HCC or any other hepatic metastatic malignancy and/or PVT [12-13], advanced cirrhosis (Child-Pugh score $\geqslant 12$ ), presence of sepsis and/or multi-organ failure requiring continuous ionotropic and/or ventilatory support after fluid and blood product resuscitation, and bleeding disorders. We also excluded patients with active angina or dynamic ECG changes (ST segment depression or elevation $\geqslant 2 \mathrm{~mm}$ ) precluding the use of terlipressin, GV bleeding, pregnant females, and those who refused to give consent.

\section{Study treatment}

Patients suspected to have EVB were potential study subjects; open label terlipressin (Novapressin ${ }^{\circledR}$ Curatis Pharma GmbH, Hannover, Germany and marketed by BF Biosciences/Ferozsons laboratories) was started at the emergency room as a bolus of $2 \mathrm{mg}$ and then $1 \mathrm{mg}$ was administered every $6 \mathrm{~h}$, for the initial $24 \mathrm{~h}$. All patients were managed in the bleeding control unit (BCU), with continuous noninvasive cardiac and hemodynamic monitoring including cardiac rhythm, pulse rate, blood pressure, and oxygen saturation. Hemoglobin $(\mathrm{Hb})$ was checked every $6 \mathrm{~h}$ for the initial $48 \mathrm{~h}$ and then every $12 \mathrm{~h}$ till discharge. Likewise, serum creatinine was checked daily. Packed red blood cells were transfused to maintain target $\mathrm{Hb}$ of $\geqslant 8 \mathrm{~g} / \mathrm{dl}$. Patients were started on IV omeprazole $40 \mathrm{mg}$ qd and switched to oral preparation once able to tolerate orally, after EVBL [14]. All patients received prophylactic intravenous ceftriaxone $2 \mathrm{~g}$ daily for three days; antibiotics were stopped if there was no other indication to continue [15]

Endoscopic variceal band ligation was performed in all patients within $12 \mathrm{~h}$ of admission, using the Saeed Six Shooter Multi-Band Ligator ${ }^{\circledR}$ (Wilson-Cook Medical, North Carolina, USA) attached to a video endoscope (Olympus GIF H-180 Tokyo, Japan). Attending gastroenterologists with at least 5 years of experience performed all EVBL.

After confirmation of EVB and successful initial hemostasis with emergency EVBL, patients were randomly assigned to continuation of terlipressin, either as SC or UC therapy, administered in a double blind dummy controlled fashion. The allocation of drug assignment was done by a pharmacist using computer generated simple random sequence at the central pharmacy of the hospital. Therefore, during the initial $24 \mathrm{~h}$ of admission, terlipressin was dispensed to both groups as an open label drug from the pharmacy. Subsequently, those random- ized to the 72-h group (UC regimen), after the initial $24 \mathrm{~h}$ of open label drug, received "active terlipressin" at a dose of $1 \mathrm{mg}$ every $6 \mathrm{~h}$ for the following $48 \mathrm{~h}$, in a $5 \mathrm{ml}$ pre-filled syringe. On the other hand, patients randomized to the 24-h arm (SC), after the initial $24 \mathrm{~h}$ of open label terlipressin, received the "terlipressin dummy" containing $5 \%$ dextrose water administered every 6 -h intervals for the following $48 \mathrm{~h}$, in a $5 \mathrm{ml}$ pre-filled syringe. Case report forms were filled daily and blood requirement, adverse events and the criteria to characterize failure to control EVB or any evidence of re-bleeding were noted.

All patients were kept at the hospital for at least 4 days from the index bleed and were then considered discharged if no other reason was observed to keep them at the hospital. At discharge, all patients were started on propranolol if there was no contraindication. They were given instructions to report to ER if they noticed any melena or hemetemesis. All patients discharged at day 4 were contacted by phone at day 5 , to assess failure to control EVB. Patients were seen 15 and 30 days after the index bleed to assess secondary outcomes. If a patient missed a follow-up appointment, they were contacted by phone to ascertain the secondary outcomes.

Endpoints

The primary endpoint was efficacy of SC to prevent failure to control EVB; failure to control EVB was defined according to the Baveno IV consensus conference guidelines [5]. According to Baveno IV, the time frame for the failure to control bleeding is any re-bleeding within $120 \mathrm{~h}$ ( 5 days). The criteria defining "failure to control EVB" were; either fresh hematemesis $\geqslant 2 \mathrm{~h}$ after start of a specific drug treatment or therapeutic endoscopy, aspiration of greater than $100 \mathrm{ml}$ of fresh blood in patients who had a naso-gastric tube in place; $\mathrm{a} \geqslant 3 \mathrm{~g}$ drop in $\mathrm{Hb}$ if no transfusion was administered, or death within 5 days. The secondary outcomes were 30-day mortality, 30-day re-bleeding defined as occurrence of new hematemesis or melena after a period of $120 \mathrm{~h}$ (5 days) attributable to esophageal varices, and 30-day composite outcome (re-bleed or death). All patients were followed for 30 days as per initial protocol but data was available for 45-day follow-up, which was not different than 30 days secondary outcomes.

\section{Statistical analysis}

We postulated that $24 \mathrm{~h}$ of terlipressin treatment is non-inferior to $72 \mathrm{~h}$ of therapy for the control of EVB. The latter regimen has been shown to reduce re-bleeding rate from $30 \%$ to $15 \%$ [16]. To demonstrate this, a sample size of 65 for the experimental group ( $24 \mathrm{~h}$ terlipressin) and 65 for the standard group ( $72 \mathrm{~h}$ terlipressin) was calculated to achieve $80 \%$ power at a $5 \%$ significance level, using a one-sided equivalence test of proportions when the proportion in the standard group is $12 \%$ and the proportion in the experimental group being tested for equivalence is $12 \%$, and the maximum allowable difference between these proportions that still results in equivalence (the range of equivalence) is $15 \%$.

All patients who had been randomly assigned to a treatment group were included in the intention-to-treat (ITT) analyses. Results are expressed as mean \pm standard deviation, median with ranges for all continuous variables and numbers (percentage) for categorical data. Univariate analysis was performed using the independent Student's $t$-test for continuous variables, and Pearson Chisquare test or Fisher exact tests were applied wherever appropriate. For the assessment of outcome measures all p-values were one-sided. Kaplan-Meier survival curves were plotted and mean bleed free survival with confidence intervals $(\mathrm{CI})$ were reported using log-rank test to assess the difference in the 30-day re-bleeding, 30-day mortality and 30-day failure to control EVB (composite outcome).

\section{Results}

\section{Study patients and study drugs}

During the study period, a total of 267 cirrhotic patients with hematemesis and/or melena were admitted. Twenty-six patients were excluded due to ischemic heart disease and were managed with adjuvant octreotide infusion. The remaining 241 patients were started on terlipressin immediately at the time of admission from the emergency room. One hundred and eleven patients were excluded from the trial after initial endoscopy as they did not meet the enrolment criteria, were considered high risk variceal bleeders or refused to give consent for the trial (Fig 1). 


\section{JOURNAL OF HEPATOLOGY}

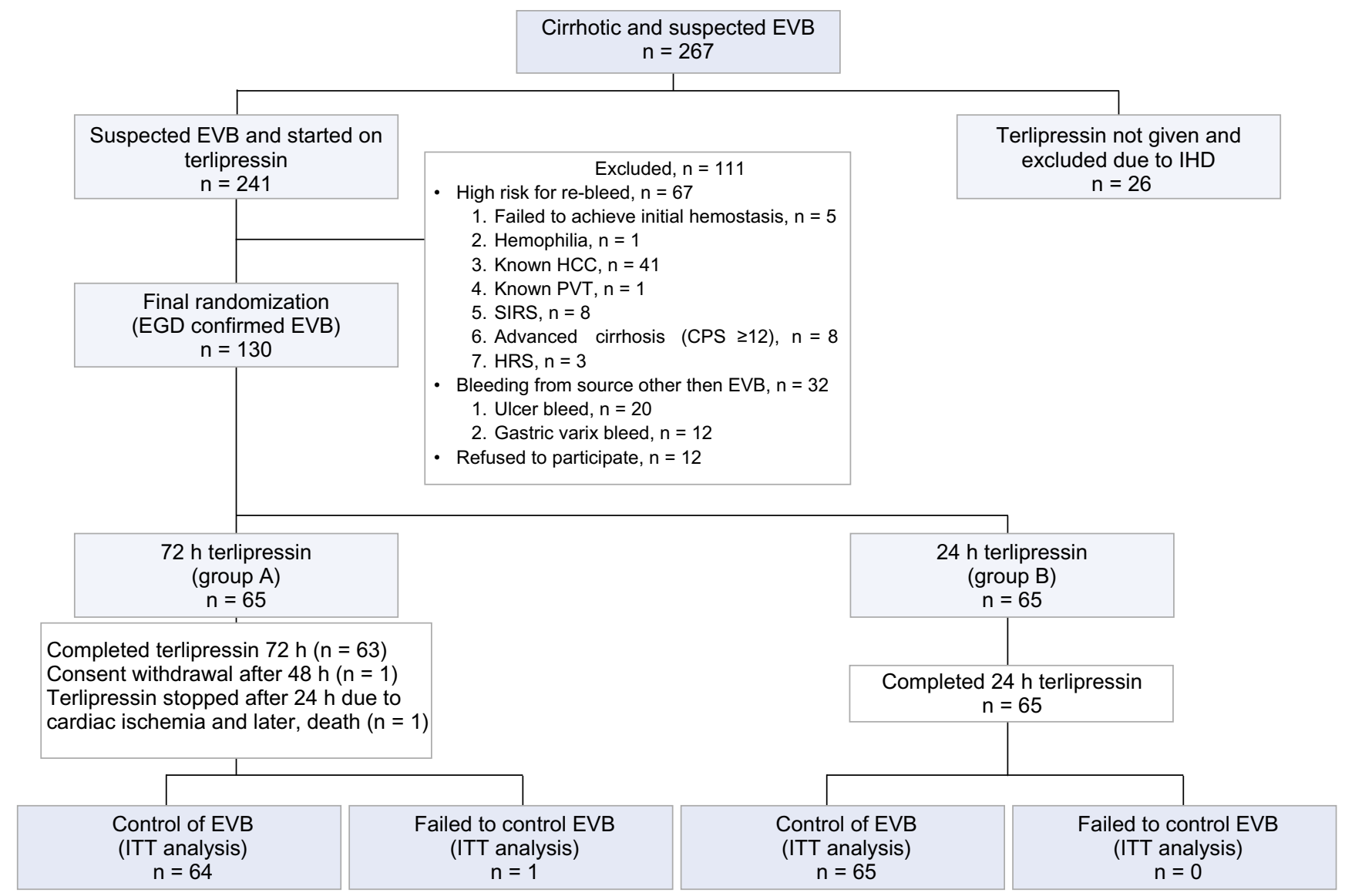

Fig. 1. Flow chart of study algorithm, enrolment, and randomization.

Finally, 65 patients were randomized to each group to receive a total of either $72 \mathrm{~h}$ (UC) or $24 \mathrm{~h}$ (SC) of terlipressin therapy. The two groups were not significantly different in their baseline characteristics; the study subjects were predominantly male and HCV was the major etiology for liver cirrhosis in both groups (Table 1).

\section{Efficacy of the 24-h terlipressin regimen}

The primary outcome was assessed for variceal re-bleeding in the initial $120 \mathrm{~h}$ ( 5 days), as defined by the Baveno IV guidelines $[5,17]$. There was only one failure to control EVB observed in the $72 \mathrm{~h}$ (UC regimen) and none in the $24 \mathrm{~h}$ of terlipressin regimen. This failure was due to the death of the patient on day 4 , due to myocardial infarction. Hence on ITT, there was no significant difference in the 72-h (UC) as compared to the 24-h (SC) treatment for the control of EVB, $p=0.50$ (Fisher exact test; 1 sided).

For the assessment of secondary outcomes, the study subjects were followed for 30 days from the index bleed (Table 2 and Fig. 2). One patient in the 72-h terlipressin group and two patients in the 24-h group had a re-bleeding within 30 days ( $p=0.50$ Fisher exact test). The mean bleeding free survival was 29.8 days (CI: 29.5-30.1) and 29.5 days (CI: 28.8-30.2) in the 72 - and 24 -h terlipressin group, respectively ( $p=0.285$, log-rank test). The all cause 30 -day mortality was 12,6 patients, $(9.2 \%)$ in each group ( $p=0.50$, Pearson Chi-square test). The mean survival was 28.4 days (CI: 27.1-29.7) and 28.2 days (CI: 26.8-29.6) in 72 - and 24 -h terlipressin group, respectively ( $p=0.495$, log-rank test).

The composite outcome of 30-day failure to control EVB, i.e. 30 days re-bleeding and/or mortality, was observed in 14 patients, seven in each group. The mean bleeding free survival was 28.3 days ( $\mathrm{CI}: 26.9-29.6$ ) and 28.1 days (CI: 26.6-29.5) in UC and SC terlipressin, respectively ( $p=0.494$, log-rank test; 1 sided). Hence, $24 \mathrm{~h}$ of terlipressin treatment was non-inferior to $72 \mathrm{~h}$ of terlipressin treatment for 30-day re-bleeding, mortality and composite outcome of failure to control EVB, when plotted on a survival curve (Table 2).

\section{Safety of the 24- $h$ terlipressin regimen}

The failure to control EVB was also the safety measure for the short course (24 h of terlipressin). As mentioned above, $24 \mathrm{~h}$ of terlipressin treatment was non-inferior to $72 \mathrm{~h}$ terlipressin treatment for the control of EVB ( $p=0.50$, Fisher exact test; 1 -sided) and was considered safe.

There was only one patient in the UC who had no history of ischemic heart disease (IHD) and a normal baseline ECG but later developed ECG changes with a troponin leak after $24 \mathrm{~h}$ of terlipressin therapy. The patient was switched to IV octreotide and died on day 4 of the index bleed due to myocardial infarction and congestive cardiac failure. Another patient in the UC group, 


\section{Research Article}

Table 1. Baseline patient characteristics of the 72- and 24-h terlipressin groups.

\begin{tabular}{|c|c|c|c|}
\hline Characteristics & $72 \mathrm{~h}$ terlipressin $(n=65)$ & $24 \mathrm{~h}$ terlipressin $(n=65)$ & $p$ values \\
\hline Age (yr) & $49.7 \pm 12.1$ & $49.8 \pm 11.2$ & 0.89 \\
\hline Gender (M) & $49(75.4 \%)$ & $48(73.8)$ & 0.84 \\
\hline $\mathrm{Hb}$ at presentation $(\mathrm{g} / \mathrm{dl})$ & $9.4 \pm 2.3$ & $9.4 \pm 2.4$ & 0.77 \\
\hline Platelets at presentation $\left(\times 10^{6}\right)$ & $116.3 \pm 45.8$ & $115.9 \pm 56.1$ & 0.45 \\
\hline Systolic blood pressure $(\mathrm{mmHg})$ & $117.1 \pm 23.9$ & $115.0 \pm 20.9$ & 0.23 \\
\hline Pulse (beats/min) & $95.3 \pm 20.1$ & $9.8 \pm 17.45$ & 0.15 \\
\hline Serum creatinine $(\mathrm{mg} / \mathrm{dl})$ & $1.3 \pm 1.6$ & $1.1 \pm 0.5$ & 0.17 \\
\hline Serum albumin (g/dl) & $2.5 \pm 0.5$ & $2.4 \pm 0.4$ & 0.35 \\
\hline Prothrombin time (sec) & $18.5 \pm 13.6$ & $18.3 \pm 9.2$ & 0.70 \\
\hline Total bilirubin (mg/dl) & $1.8 \pm 1.3$ & $1.9 \pm 1.2$ & 0.36 \\
\hline Child-Pugh score & $8.4 \pm 1.6$ & $8.9 \pm 1.7$ & 0.52 \\
\hline Packed red cell transfusion (units) & $1.2 \pm 1.3$ & $1.1 \pm 1.0$ & 0.17 \\
\hline Active bleed & $15(20.1 \%)$ & $16(24.6 \%)$ & 0.84 \\
\hline Presence of ascites & $23(35.4 \%)$ & $25(38.5 \%)$ & 0.13 \\
\hline $\begin{array}{l}\text { Grade of varices } \\
\qquad \begin{array}{r}\text { Grade II } \\
\text { Grade III } \\
\text { Grade IV }\end{array}\end{array}$ & $\begin{array}{l}6(9.2 \%) \\
25(38.5 \%) \\
34(52.3 \%)\end{array}$ & $\begin{array}{l}9(13.8 \%) \\
25(38.5 \%) \\
31(47.7 \%)\end{array}$ & 0.69 \\
\hline $\begin{array}{l}\text { Child-Pugh Class } \\
\text { Child's A } \\
\text { Child's B } \\
\text { Child's C }\end{array}$ & $\begin{array}{l}7(10.8 \%) \\
41(63 \%) \\
17(26.2 \%)\end{array}$ & $\begin{array}{l}7(10.8 \%) \\
30(46.2 \%) \\
28(43 \%)\end{array}$ & 0.11 \\
\hline $\begin{array}{l}\text { Etiology of cirrhosis } \\
\text { HCV } \\
\text { HBV } \\
\text { Cryptogenic } \\
\text { HBV + HCV } \\
\text { Alcoholic }\end{array}$ & $\begin{array}{l}33(50.8 \%) \\
10(15.4 \%) \\
7(10.8 \%) \\
4(6.2 \%) \\
11(16.9 \%)\end{array}$ & $\begin{array}{l}42(64.6 \%) \\
6(9.2 \%) \\
10(15.4 \%) \\
3(4.6 \%) \\
4(6.2 \%)\end{array}$ & 0.19 \\
\hline
\end{tabular}

in whom bleeding was controlled, developed hemorrhagic pancreatitis of unknown etiology at the time of discharge and died on day 9 of index bleed. One diabetic patient in the 24-h terlipressin group developed acute stroke on day 6 after discharge, following completion of his trial dose. Causes of death over 30-day follow-up and possible side effects are shown in Table 3.

\section{Discussion}

The optimal duration of vasoactive drug treatment in bleeding cirrhotic patients treated with endoscopic therapy is not known. For the management of all patients with EVB, most guidelines, including Baveno IV, $[5,18]$ consider the treatment duration of terlipressin as a minimum of 3 days $(72 \mathrm{~h})$. Our study shows that 24-h terlipressin treatment is non-inferior to 72-h terlipressin treatment in acute EVB, as adjuvant therapy to successful EVBL. As far as we know, we have demonstrated for the first time, with a double blind study, the efficacy of ultra-short course (24 h) vs. $72 \mathrm{~h}$ of terlipressin treatment, as adjuvant therapy to endoscopic variceal band ligation in acute variceal bleeding. This trial has clinical implications of cost savings and may help shorten the length of hospital stay in clinical practice and probably will be associated with fewer side effects. On the basis of our previous report [11] and the results of this trial, we may recommend shortening the duration of therapy in future guidelines for the management of EVB in acute EVB patients.

There have been other recent attempts of shortening the duration of terlipressin therapy but none has compared adjuvant therapy in each arm with shortening the duration of therapy. A double blind, multi center randomized controlled trial reported no significant difference in the efficacy to control variceal bleeding in a shorter course of therapy, i.e. $48 \mathrm{~h}$ of low dose of terlipressin ( $0.2 \mathrm{mg}$ every $4 \mathrm{~h})$ vs. $1 \mathrm{mg}$ every $4 \mathrm{~h}$, for 5 days [19]. Terlipressin was, however, used as monotherapy rather than as an adjunct to EVBL, and perhaps because of these reasons the 30 -day mortality was reported as $17.1 \%$ in the 48 -h therapy group and $20 \%$ in the 5-day therapy group [19]. In contrast, in our trial, the 30 -day mortality was $9.2 \%$ in both groups, this was due to the fact that our study subjects comprised patients in whom we had achieved initial hemostasis with EVBL. Patients at high risk for re-bleeding were excluded and we used terlipressin as adjuvant therapy to EVBL, as recommended by most clinical guidelines.

In another recent study, $48 \mathrm{~h}$ of terlipressin (SC) treatment along with EVBL, in low risk EVB patients, was compared with terlipressin alone for 5 days [20]. This was a superiority trial comparing a shorter course of adjuvant terlipressin to the standard 5 days of terlipressin monotherapy and showed that even in 


\section{JOURNAL OF HEPATOLOGY}

Table 2. Secondary outcome measures.

\begin{tabular}{|c|c|c|c|}
\hline & $72 \mathrm{~h}$ terlipressin & $24 \mathrm{~h}$ terlipressin & $p$ value \\
\hline $\begin{array}{l}30 \text { days re-bleed } \\
\qquad \text { (Mean days with } 95 \% \mathrm{Cl} \text { ) }\end{array}$ & $29.8(29.5-30.1)$ & $29.5(28.8-30.2)$ & $0.285^{*}$ \\
\hline No. with proportion & $1(1.5 \%)$ & $2(3.1 \%)$ & $0.500^{\dagger}$ \\
\hline $\begin{array}{l}30 \text { days mortality } \\
\text { (Mean days with } 95 \% \mathrm{Cl} \text { ) }\end{array}$ & $28.4(27.1-29.7)$ & $28.2(26.8-29.6)$ & $0.495^{*}$ \\
\hline No. with proportion & $6(9.2 \%)$ & $6(9.2 \%)$ & $0.500^{\ddagger}$ \\
\hline $\begin{array}{l}\text { Composite outcome (30 days failure to control EVB) } \\
\text { (Mean days with } 95 \% \mathrm{Cl} \text { ) }\end{array}$ & $28.4(27.0-29.8)$ & $28.2(26.7-29.7)$ & $0.495^{*}$ \\
\hline No. with proportion & $7(10.8 \%)$ & $7(10.8 \%)$ & $0.500^{\ddagger}$ \\
\hline
\end{tabular}

${ }^{*}$ Log-rank test (1-sided)

${ }^{\dagger}$ Fisher exact test (1-sided).

"Pearson's Chi-square test (1-sided).

Table 3. Possible cause of the 30-day mortality and side effects.

\begin{tabular}{lll}
\hline A & \multicolumn{3}{c}{ Terlipressin } \\
Cause of death & (UC) $72 \mathrm{~h}$ & (SC) 24 h \\
\hline Myocardial infarction & 1 & 0 \\
Sepsis and SBP & 2 & 1 \\
Re-bleed & 0 & 1 \\
PSE and aspiration pneumonia & 1 & 1 \\
COPD exacerbation & 0 & 1 \\
Pancreatitis & 1 & 0 \\
Stroke & 0 & 1 \\
Duodenal ulcer bleed & 1 & 0 \\
Unknown (found dead) & 0 & 1 \\
\hline B & & Terlipressin \\
Adverse events (side effects) & $($ UC) $72 \mathrm{~h}$ & $(\mathrm{UC}) 72 \mathrm{~h}$ \\
\hline Myocardial infarction & 1 & 0 \\
Pancreatitis & 1 & 0 \\
Stroke & 0 & 1 \\
\hline
\end{tabular}

low risk EVB, the combination of EVBL and terlipressin was superior to terlipressin alone. On the other hand, our trial was a noninferiority trial addressing a similar EVB patients' group and we could confirm that the duration of adjuvant terlipressin therapy could be ultra-shortened to $24 \mathrm{~h}$ and this was as effective as the UC adjuvant terlipressin treatment.

High serum creatinine and bilirubin levels, and the presence of encephalopathy are considered to be independent factors predictive of re-bleeding and mortality, as reported by D'Amico et al. [2] and according to our own retrospective variceal bleeding data base source analysis [21]. We therefore took the arbitrary cut off of Child-Pugh score of 12 to cover these factors and defined these patients as high risk and excluded them from the trial. This most likely explains the high success of EVB control in the current trial, along with the fact that terlipressin was used as an adjuvant to EVBL. Additionally, we believe that the high success rate achieved with the treatment is partly due to the fact that we have included in the trial only those patients in whom successful variceal band ligation was performed and, immediately after endoscopy, we randomized them to receive ultra-short course (total $24 \mathrm{~h}$ ) vs. usual course (total $72 \mathrm{~h}$ ) of terlipressin.

Being a tertiary referral center, we may be experiencing a referral bias towards patients who are able to reach our center from various distances due perhaps to the fact that they may have a high tendency to stop bleeding spontaneously. However, we believe that this unexpected bias does not alter our study results. There were 25\% active bleeders in both groups (fresh blood in naso-gastric tube or active esophageal spurting seen at endoscopy) and $75 \%$ had melena at presentation but were not actively bleeding, as shown in Table 1 . Therefore, the number of patients who were still actively bleeding at presentation and in whom bleeding had stopped spontaneously was not different between the two treatment arms.

In our hands, the overall safety of terlipressin was comparable to that reported in other studies, as we encountered only one patient who developed ECG changes and non-ST elevation, myocardial infarction and later died. One patient in the 72-h treatment regimen developed hemorrhagic pancreatitis on the day of planned discharge and later died. The cause and effect relationship of terlipressin in causing ischemic pancreatitis remains unclear to us and could well be a chance finding. No re-bleeding was observed in both patients who died while in the trial, possibly due to terlipressin side effects.

We observed only one failure to control bleeding in the usual course terlipressin on ITT, but none for the protocol analysis. This low outcome was a limitation of the study design as our aim was to assess the group with shorter duration where the patients had achieved initial variceal bleeding control with EVB. Hence, our results cannot be generalized to all EVB patients and are applicable to only EVB subjects achieving haemostasis with EVBL, in whom we believe the duration of terlipressin therapy can be safely and effectively shortened to $24 \mathrm{~h}$ only.

In conclusion, short course terlipressin as an adjuvant to EVBL is as effective as UC for the control of EVB; all cause 30-day mortality, 30-day re-bleeding and composite outcome of 30-day failure to control EVB were not different when terlipressin was used for $24 \mathrm{~h}$ in these patients. A larger population sample or metaanalysis studies are needed to better confirm the present results.

\section{Financial support}

The research team acknowledges the unconditional support of Ferozsons Laboratories (BF Bio-Sciences), Pakistan to support this 
A

B
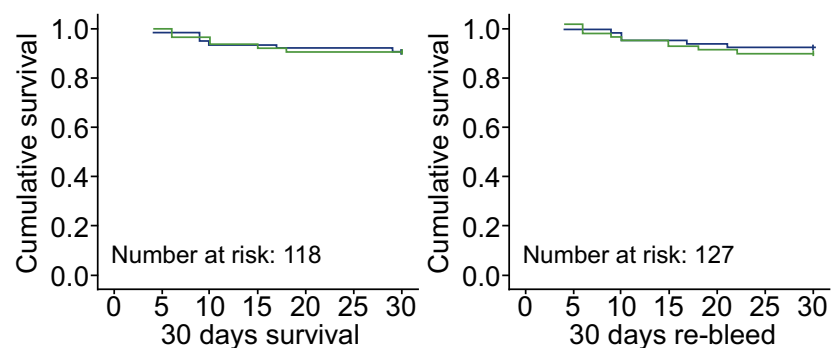

C

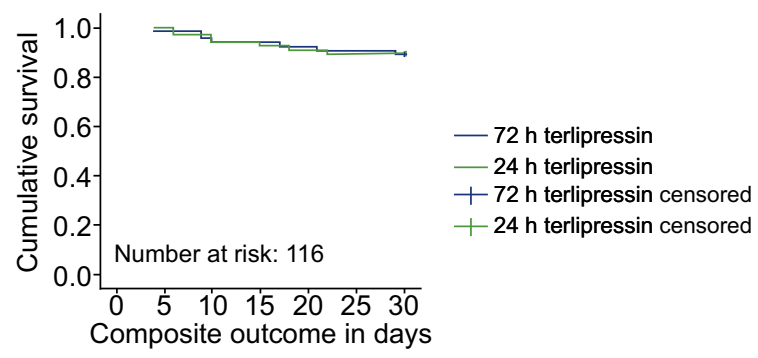

Fig. 2. Kaplan-Meier graphs of $\mathbf{3 0}$ day survival, re-bleeding and composite outcome. (A) 30-day survival, (B) re-bleeding, and (C) composite outcome.

investigator-initiated clinical trial in terms of bearing the cost of terlipressin, clinical research associate honorarium, and pharmacy charges for the generation of randomization sequence, blinding, placebo preparation, and dispensing of investigational products. The study was an investigator-initiated clinical trial and the protocol was written by the investigators, the study was performed by the research team and the subject, care givers, investigator, and outcomes assessor were blinded for the study assignment. The study sponsor (BF Biosciences/Ferozsons Laboratories, Pakistan) had no role in the study design, in the collection, analysis, and interpretation of data.

\section{Author contribution}

Zahid Azam and Saeed Hamid contributed in terms of original idea, study design, protocol writing, organization of logistics, funding, and article editing. Zahid Azam additionally contributed to the statistical analysis, review, and submission of the final manuscript. Wasim Jafri and Shahab Abid contributed to study design, logistics, care for patients under study, and article editing. Mohammed Salih contributed to the coordination between various departments, literature search, article writing with 1st author. Hasnain Ali Shah and Zaigham Abbas contributed to study design, care of study patients, and editing of the article.

\section{Guarantor of the article}

Ethics Review Committee and Professor Saeed Hamid (Corresponding author), Chair Department of Medicine Aga Khan University.

\section{Conflict of interest}

This work was supported by an unrestricted Grant from Ferozsons Laboratories (BF Bio-Sciences), Pakistan.

\section{References}

[1] Grace ND. Diagnosis and treatment of gastrointestinal bleeding secondary to portal hypertension. American College of Gastroenterology Practice Parameters Committee. Am J Gastroenterol 1997;92:1081-1091.

[2] D'Amico G, De Franchis R. Upper digestive bleeding in cirrhosis. Post therapeutic outcome and prognostic indicators. Hepatology 2003;38:599-612.

[3] Carbonell N, Pauwels A, Serfaty L, Fourdan O, Levy VG, Poupon R. Improved survival after variceal bleeding in patients with cirrhosis over the past two decades. Hepatology 2004;40:652-659.

[4] Chalasani N, Kahi C, Francois F, Pinto A, Marathe A, Bini EJ, et al. Improved patient survival after acute variceal bleeding: a multicenter, cohort study. Am J Gastroenterol 2003;98:653-659.

[5] de Franchis R. Evolving consensus in portal hypertension. Report of the Baveno IV consensus workshop on methodology of diagnosis and therapy in portal hypertension. J Hepatol 2005;43:167-176.

[6] Dell'Era A, de Franchis R, Iannuzzi F. Acute variceal bleeding: pharmacological treatment and primary/secondary prophylaxis. Best Pract Res Clin Gastroenterol 2008;22:279-294.

[7] Ioannou G, Doust J, Rockey DC. Terlipressin for acute esophageal variceal hemorrhage. Cochrane Database Syst Rev 2003;1:CD002147.

[8] Ioannou GN, Doust J, Rockey DC. Systematic review: terlipressin in acute oesophageal variceal haemorrhage. Aliment Pharmacol Ther 2003;17:53-64.

[9] Lebrec D. A discussion of how terlipressin limits mortality in cases of bleeding oesophageal varices. Eur J Gastroenterol Hepatol 1998;10:549-552.

[10] Abid S, Jafri W, Hamid S, Salih M, Azam Z, Mumtaz K, et al. Terlipressin vs. octreotide in bleeding esophageal varices as an adjuvant therapy with endoscopic band ligation: a randomized double-blind placebo-controlled trial. Am J Gastroenterol 2009;104:617-623.

[11] Azam Z, Hamid S, Jafri W. Efficacy of short course of terlipressin as an adjuvant therapy for the control of acute variceal bleed. Hepatology 2008;48:[abstract no 721].

[12] Lang BH, Poon RT, Fan ST, Wong J. Outcomes of patients with hepatocellular carcinoma presenting with variceal bleeding. Am J Gastroenterol 2004;99:2158-2165.

[13] Kadouchi K, Higuchi K, Shiba M, Okazaki H, Yamamori K, Sasaki E, et al. What are the risk factors for aggravation of esophageal varices in patients with hepatocellular carcinoma? J Gastroenterol Hepatol 2007;22:240-246.

[14] Shaheen NJ, Stuart E, Schmitz SM, Mitchell KL, Fried MW, Zacks S, et al. Pantoprazole reduces the size of postbanding ulcers after variceal band ligation: a randomized, controlled trial. Hepatology 2005;41:588-594.

[15] Hou MC, Lin HC, Liu TT, Kuo BI, Lee FY, Chang FY, et al. Antibiotic prophylaxis after endoscopic therapy prevents rebleeding in acute variceal hemorrhage: a randomized trial. Hepatology 2004;39:746-753.

[16] D'Amico G, Pietrosi G, Tarantino I, Pagliaro L. Emergency sclerotherapy versus vasoactive drugs for variceal bleeding in cirrhosis: a Cochrane metaanalysis. Gastroenterology 2003;124:1277-1291.

[17] Bureau C, Peron JM, Alric L, Morales J, Sanchez J, Barange K, et al. "A La Carte" treatment of portal hypertension: adapting medical therapy to hemodynamic response for the prevention of bleeding. Hepatology 2002;36:1361-1366.

[18] Farooqi JI, Jafri SM, Najib ul H, Niaz SK, Hamid S, Abbas Z, et al. Management of variceal bleeding: PSG guidelines 2006. J Pak Med Assoc 2007;57:505-511.

[19] Bruha R, Marecek Z, Spicak J, Hulek P, Lata J, Petrtyl J, et al. Double-blind randomized, comparative multicenter study of the effect of terlipressin in the treatment of acute esophageal variceal and/or hypertensive gastropathy bleeding. Hepatogastroenterology 2002;49:1161-1166.

[20] Lo GH, Chen WC, Wang HM, Lin CK, Chan HH, Tsai WL, et al. Low-dose terlipressin plus band ligation versus low-dose terlipressin alone in the prevention of very early rebleeding of oesophageal varices. Gut 2009;58:1275-1280.

[21] Ismail FW, Mumtaz K, Shah HA, Hamid S, Abbas Z, Abid S, et al. Factors predicting in-hospital mortality in patients with cirrhosis hospitalized with gastro-esophageal variceal hemorrhage. Indian J Gastroenterol 2006;25:240-243. 